\title{
Robust Signals Detection Algorithm Based on Cyclostationarity in Impulsive Noise
}

\author{
Shun Na, Penghui Li, Jing Zhang, Yang Liu, Yong Tie* and Yongjun Jia \\ Received: January 1, 2020. Revised: March 20, 2020. 2nd Revised: May 26, 2020. \\ Accepted: June 18, 2020. Published: July 24, 2020.
}

\begin{abstract}
A robust method for detecting the communication signals impinging on an antenna with interference and non-Gaussian impulsive noise is introduced in this paper. Degradation of the conventional cyclic detector which based on max-output-SNR criterion in impulsive noise is shown both theoretically and experimentally. By fusing second-order cyclostationarity and fractional lower-order statistics, a type of cyclic fractional lower-order statistics is developed which is defined for exploiting cyclostationarity property. Then, a new robust type of detection algorithm is developed using the theory of optimal filtering based on max-output-SNR criterion and alpha-stable distribution, including the fractional lower-order cyclic matched filter, which is formulated for detecting the communication signals in the presence of interference and non-Gaussian alpha-stable distribution impulsive noise. It is shown that the new method is robust to Gaussian and non-Gaussian impulsive noises, and is immune to the interfering signals which occupy the same spectral band as that of the received signal. Simulation results show the robustness and effectiveness of the proposed algorithm.
\end{abstract}

Keywords-Alpha stable distribution, Cyclostationarity, Fractional lower-order statistics, Impulsive noise, Signal detection

\section{INTRODUCTION}

$\mathrm{T}$ HE need to detect the signals impinging on an array of sensors arises naturally in the wireless communication networks, radar, and sonar systems [1]. In many approaches to the detection problem for communication signals, max-output-signal-to-noise is adopted as the detection criterion. In the light of the growing use of spread-spectrum techniques and the increasingly congested communication environments standard method of signal detection, which is based on the max-output-signal-to-noise criterion, is referred to as matched

This work is supported in part by the Program for Young Talents of Science and Technology in Universities of Inner Mongolia Autonomous Region under Grant NJYT-20-A11, and in part by the Natural Science Foundation of Inner Mongolia Autonomous Region under Grant 2019MS06033 and by National Key R\&D Program of China under Grant 2016YFC1401004.

Shun $\mathrm{Na}$, Penghui $\mathrm{Li}$, and Jing Zhang is with the College of Electronic Information Engineering, Inner Mongolia University, Hohhot, 010021, China.

Yang Liu is with the College of Electronic Information Engineering, Inner Mongolia University, Hohhot, 010021, China; and is with the School of Electronics and Computer Science, University of Southampton, Southampton, UK, SO171BJ.

Yong Tie is with the College of Electronic Information Engineering, Inner Mongolia University, Hohhot, 010021, China. (Corresponding author to provide E-mail: tieyongs@163.com).

Yongjun Jia is with the National Satellite Ocean Application Service, Beijing, 100081, China. filter. Matched filter is the optimum receiver for an additive white Gaussian noise channel. It essentially takes advantages of the fact that the signal frequency components are coherent in nature whilst the corresponding noise components are incoherent. It is possible, using appropriate processing, to add spectral components of the signal voltage-wise whilst the same processing adds noise components only power-wise. Matched filter is widely applied in communication signal detection, parameter estimation, and system identification [2]. In view of the tremendous diversity of theory, analytical technique, and method of implementation in the field of filtering, the objective of filtering seems almost trivial: viewed in the time-domain, the objective is to convolve a signal with impulse-response function, which means to add up weighted versions of delayed replicas of the signal. Viewed in the frequency domain, the objective is to multiply the spectral components of the signal by a transfer function, which means to scale their strengths and shift their phase. In general, since matched filtering method simply measures energy in specific bands of frequency, it is inherently susceptible to unknown or changing background noise level and interference. In order to design secure communication systems, it is necessary to assess the vulnerability of competing techniques to signal detection [3].

Communication signals have traditionally been modeled as stationary random processes. Although communication signals typically involve one or more periodicities underlying their random fluctuations, due to sine-wave carriers and repetitive pulsing or keying, a stationary model can be obtained by introducing random phase variables uniformly distributed over on period of each periodicity. Although in some cases these periodicities can be ignored by signal processors, such as receivers which must detect the presence of signals of interest, estimate their parameters, and extract their messages, in many cases they can be much to gain in terms of improvements in performance of these signal processors by recognizing and exploiting underlying periodicity. This typically requires that the random signal be modeled as cyclostationary, in which cases the statistical parameters vary in time with single or multiple periodicities [4], [5]. By exploiting the periodicity property or cyclostationarity of communication signals, the problem of optimally filtering the modulated signals was discussed in [6], [7], and the cyclic matched filter is proposed in [6]. It has been demonstrated that, in comparison with the optimum detector for the stationary model of the signal, superior detection 
performance can be obtained by exploiting the single frequency of some harmonics of a cyclostationarity, such as a double carrier frequency, or a keying rate. It has also been demonstrated in [8] that, almost of cyclostationary signals in communication networks have more than on cycle frequency, in comparison with the single cycle cyclic matched filter, superior performance can be obtained by the cyclic matched filter bank which exploits more than one cycle frequency.

The matched filtering and cyclic matched filtering theories are proposed under the assumption that the environment noise is assumed to be Gaussian distributed with finite second-order statistics. As a result of the Gaussian assumption, both of the two methods are based on the second-order statistics of signals. Generally, it is reasonable to assume that the noise is Gaussian distribution, because it may lead to closed-form solutions. However, in some applications such as communication networks, radio astronomy, radar system, etc., the noise has impulsive effects in short time [9-11]. In these scenarios, it is appropriate to model the noise as non-Gaussian distribution. We are interested in developing signal detection methods for a large of class of random processes which include the Gaussian process as a special element. Studies and experiments results show that the class of alpha-stable distributions is better for modeling impulsive noise than Gaussian distribution in real life applications [12-16]. By the generalized central limit theorem, they are the only class of distributions that can be the limit distributions for sums of i.i.d. random variables [16]. Another important property of the stable laws is the stability property. Gaussian is the limiting case with $\alpha=2$, and Cauchy distribution has $\alpha=1$. The alpha-stable distributions can model some types of noise such as atmospheric noise, underwater noise very well. Therefore, developing robust signal detecting techniques using the alpha-stable model is important both in theory and in practice. As stable distribution does not have finite second-order moment (except for $\alpha=2$ ), or even first-order moment $(\alpha<2)$ [21], both conventional matched filter and cyclic matched filter will be considerably weakened in stable distribution impulsive noise environments. The robust filtering methods of signals which outperform effects of impulsive noise are introduced in [18-20]. These detectors based on fractional lower-order statistics are robust to both Gaussian and impulsive noise. However, the receivers in communications networks are subject to a variety of types of electromagnetic interference. These interfering signals may occur at the same time and occupy the same frequency band as that of the signal of interest, can severely degrade the performance of conventional signal detectors.

The purpose of this paper is to present a new signal detection method that exploits an inherent signal property, called fractional lower-order cyclostationarity that is characteristic of manmade signals used in communication systems to obtain substantial tolerance to some types of interference, Gaussian and impulsive noises. We consider modulated signals, which are used for spread-spectrum communications, and we determine receiver operating characteristics for operation in a relatively strong alpha-stable impulsive noise background. We combine second-order cyclostationarity with fractional lower-order statistics to form a new type of fractional-lower order cyclostationary statistics, which can exploit the spectral correlation property of cyclostationary signals in the presence of impulsive noise. A new robust cyclic matched filtering method for cyclostationary signals is proposed in this paper. The new cyclic matched filter employs fractional-lower order cyclostationarity to robust against the interfering signals, Gaussian noise, and impulsive noise. Like most conventional optimal detectors, the new robust method is based on max-output-SNR criterion. However, unlike conventional methods, the new detector exploits cyclostationarity property using fractional lower-order cyclic statistics. It has the advantages of both FLOS based filtering methods and cyclic matched filter. Simulation results demonstrate that the proposed matched filtering method can acquire higher accuracy of signal detection than that of the conventional cyclic matched filter in the presence of interference and impulsive noise.

The rest of this paper is organized as follows: The fundamental of alpha-stable distribution is briefly described in Section 2. In Section 3, the cyclic correlation matched filter is described. A new robust optimal filtering method based on fractional lower-order cyclic statistics is proposed in section 4 . In section 5, the performance of the new algorithm via Monte Carlo simulations is presented. Finally, conclusions are given in Section 6.

\section{FundAMENTALS OF ALPHA-STABLE DiSTRIBUTION}

Stable processes satisfy the stability property which demonstrates that linear combinations of jointly stable variables are stable. They arise as limiting processes of sums of independent, identically distributed random variables via the generalized central limit theorem. An alpha-stable process can be described conveniently by its characteristic function [9], [10]:

$\varphi(t)=\exp \left(j a t-\gamma|t|^{\alpha}[1+j \beta \operatorname{sign}(t) \omega(t, \alpha)]\right)$

where $\omega(t, \alpha)=\tan (\alpha \pi / 2)$ if $\alpha \neq 1$ and $(\pi / 2) \log |t|$ if $\alpha=1$; in addition $-\infty<a<\infty, \gamma>0,0<\alpha \leq 2,-1 \leq \beta \leq 1$. A stable distribution is completely determined by four parameters, here $\alpha$ is the characteristic exponent, which controls the thickness of the tail in the distribution. The Gaussian process is a special case of stable process with $\alpha=2$. The dispersion parameter $\gamma$ is similar to the variance of Gaussian process, and $\beta$ is the symmetric parameter. When $\beta=0$, the distribution is symmetric and the observation is referred to as the $S \alpha S$ (symmetric $\alpha$-stable) distribution. When $\alpha=2$ and $\beta=0$, the stable distribution becomes the Gaussian distribution and $\gamma=\sigma^{2} / 2$.

The main difficulty in developing signal processing methods based on stable processes is that the variable space is not a Hilbert space as in the case of Gaussian process, but a Banach space $(1<\alpha<2)$ or a Metric space $(0<\alpha \leq 1)$ both of which 
are more unyielding in their structure. Stable distributions do not have finite second-or higher order moments and even first-order moment [21]. The fractional lower-order statistics become the new tools for signal processing. Covariation and fractional lower-order covariance (FLOC) play the roles analogous to the covariance. For two jointly random $S \alpha S$ variables $X$ and $Y$, covariation is defined as $[X, Y]_{\alpha}=\int_{S} x y^{\langle\alpha-1\rangle} \mu(d s)$, where $S$ is the unit circle, and $\mu(\cdot)$ is the spectral measure of the $S \alpha S$ random vector $(X, Y)$, and convention $Z^{\langle p\rangle}=|Z|^{p+1} / Z$ for any real or complex number $Z$. Covariation can be computed using the fractional lower-order moment (FLOM)

$\operatorname{COV}[X, Y]=\frac{E\left(X Y^{\langle p-1\rangle}\right)}{E\left(|Y|^{p}\right)} \gamma_{y}$

Fractional lower-order covariance is defined as

$\operatorname{FLOC}[X, Y]=\frac{E\left(X^{\langle p-1\rangle} Y^{\langle p-1\rangle}\right)}{E\left(|Y|^{p}\right)} \gamma_{y}$

The basic properties of the covariation are summarized in [10], for any $\eta_{1}$ and $\eta_{2}$, we have $\left\langle a \eta_{1}+b \eta_{2}, \varsigma\right\rangle_{p}=$ $a\left\langle\eta_{1}, \varsigma\right\rangle_{p}+b\left\langle\eta_{2}, \varsigma\right\rangle_{p}$. If $\eta_{1}$ and $\eta_{2}$ are independent, then $\left\langle\eta_{1}, \eta_{2}\right\rangle_{p}=0$, while the converse is not true. The FLOS of a random process have been found useful in designing algorithms that exhibit resistance to outliers and allow for robust processing of impulsive, as well as Gaussian data. This property of FLOS-based algorithms will be introduced for the communication signals detection problem addressed in this paper.

\section{CyClic CORRElation MATCHED Filtering Method}

The theory of matched filtering was first proposed by D. D. North in 1943. A signal impinging on an antenna element of receiver, together with interfering signals and received noise, can be modeled as

$x(t)=s(t)+n(t)$

where $s(t)$ is the signal of interest (SOI), $n(t)$ and $m(t)$ are the signals not of interest (SNOI), including interference and noise. For the received signal $x(t)$, the transfer function of maximum output SNR matched filter is given by

$H_{\text {opt }}(f)=\frac{S^{*}(f)}{P_{n}(f)} e^{-j 2 \pi f T_{0}}$

where $S(f)=\int s(t) e^{-j 2 \pi f t} d t$ is the spectrum of SOI, $P_{n}(f)$ is the power spectrum of SNOI,

$P_{n}(f)=\left|\int n(t) e^{-j 2 \pi f t} d t\right|^{2}$

and $T_{0}$ is received time delay.

In communication networks, the received signal is always corrupted by interfering signals which occur at the same time as the signal of interest and occupy the same spectral band as that of the SOI. Since $S(f)$ contains the frequency components of interference and noise, the performance of matched filter will be degraded. In order to serve potential users of cyclostationarity, the general theoretical development of optimum filtering for cyclostationary signals was presented in [7], [8] which was called cyclic matched filter. It follows that the cyclic correlation of received signal is given by

$R_{x}^{\varepsilon}(\tau)=R_{s}^{\varepsilon}(\tau)+R_{n s}^{\varepsilon}(\tau)+R_{n}^{\varepsilon}(\tau)$

where $R_{s}^{\varepsilon}(\tau)$ is defined as

$R_{s}^{\varepsilon}(\tau) \square\left\langle s(t+\tau) s^{*}(t) e^{-j 2 \pi \varepsilon t}\right\rangle$

is referred to as cyclic autocorrelation function and the frequency $\varepsilon$ is called cycle frequency. The cyclic autocorrelation function is a characteristic property of second-order periodicity, in that $s(t)$ is said to contain cyclostationarity if and only if the limit cyclic autocorrelation function is not identically zero for some nonzero cycle frequency $\varepsilon$. The cyclic spectrum is defined to be the Fourier transform of the cyclic autocorrelation.

$S_{s}^{\varepsilon}(f) \square \int_{-\infty}^{\infty} R_{s}^{\varepsilon}(f) e^{-j 2 \pi f \tau} d \tau$

If the SOI exhibits a cycle frequency $\varepsilon$ not shared by the SNOI, then by using this value of $\varepsilon$ in (7), we obtain $R_{n}^{\varepsilon}(\tau)=0$. Equation (6) will reduce to

$R_{x}^{\varepsilon}(\tau)=R_{s}^{\varepsilon}(\tau)+R_{n s}^{\varepsilon}(\tau)$

From (8) and (9) we get

$S_{x}^{\varepsilon}(f)=S_{s}^{\varepsilon}(f)+S_{n s}^{\varepsilon}(f)$

This is the means by which we obtain signal selectivity in measurements. Equations (9) and (10) indicate that ideal second-order cyclostationarity measurements are immune to interfering signals and noise which do not share the same cycle frequency as that of the SOI. Therefore, the signal processing methods based on cyclostationarity is more tolerant to the interference than processing methods based on stationary signal model.

In equation (9), we assume that $z_{x}(\tau)=R_{x}^{\varepsilon}(\tau)$, $z_{s}(\tau)=R_{s}^{\varepsilon}(\tau)$, and $m(\tau)=R_{s n}^{\varepsilon}(\tau)$, then equation (9) will yield $z_{x}(\tau)=z_{s}(\tau)+m(\tau)$

It has been demonstrated that $m(\tau)$ is a stationary process which with zero mean [8]. The power spectrum of $m(\tau)$ is

$G_{m}(f)=\frac{1}{T} S_{s}^{0}(f+\varepsilon) G_{n}(f)$

where $S_{s}^{0}(f+\varepsilon)$ is spectral correlation function of $s(t)$ as cycle frequency $\varepsilon$ equals to zero. Furthermore, from (8) we know that

$S_{z_{s}}(f)=S_{s}^{\varepsilon}(f)$

$S_{z_{x}}(f)=S_{z_{s}}(f)+G_{m}(f)$

Thus, the problem of optimally filtering of the signal model (11) is to obtain the transfer function of the matched filter that achieves the maximum SNR output when the SOI is $z_{s}(\tau)$. 
Substitution of (12)-(14) into (4), we get the transfer function of cyclic matched filter

$$
H(f)=c T\left[\left(S_{s}^{\varepsilon}(f)\right)^{*} /\left(S_{s}^{0}(f+\varepsilon) G_{n}(f)\right)\right] e^{-j 2 \pi f T_{0}}
$$

where $\mathrm{c}$ is a constant. It has been demonstrated in [4], [5] that, if the cycle frequency $\varepsilon$ is not shared by interference and noise, the ideal limit spectral correlation function of $n(t)$ equals to zero. Therefore, compared with conventional matched filter, the cyclic matched filter is immune to interference and noise which do not share the same cyclostationarity as SOI.

However, as stable processes do not have finite second-order moments, except for the limiting case $\alpha=2$ (Gaussian distribution). If $X$ be a $S \alpha S$ random variable, then $E\left[|X|^{p}\right]=\infty$ for all $p>\alpha$. If the received noise in $n(t)$ contains impulsive components, then second-order moment $E[x(t) x(t+\tau)] \rightarrow \infty$. From equation (7) we know that cyclic correlation is the Fourier coefficient of Fourier series expansion of second-order autocorrelation function. Therefore, cyclic autocorrelation function and cyclic spectrum will become infinite in impulsive noise

$$
\begin{aligned}
& R_{x}^{\varepsilon}(\tau) \square \lim _{T \rightarrow \infty} \frac{1}{T} \int_{-T / 2}^{T / 2} x(t+\tau) x^{*}(t) e^{-j 2 \pi \varepsilon t} d t=\infty \\
& S_{x}^{\varepsilon}(f) \square \int_{-\infty}^{\infty} R_{x}^{\varepsilon}(f) e^{-j 2 \pi f \tau} d \tau=\infty
\end{aligned}
$$

It indicates that second-order cyclic correlation and cyclic spectrum will not applicable to impulsive noise environment. Since second-order cyclic statistics is not applicable, performance of cyclic matched filter based on conventional spectral correlation function can also severely degrade.

\section{Robust Cyclic COMMUNICATION SignALS DETECTION ALGORITHM}

In this section, we indicate a new robust optimal filtering method for communication signals. Since second-order cyclic autocorrelation function and cyclic spectrum will not be applicable to non-Gaussian impulsive noise, it is necessary to define new tools which can be used to exploit cyclostationarity of communication signals in the presence of impulsive noise. The fractional lower-order statistics is more suitable than second-order statistics in practical signals processing applications under the additive alpha-stable noise [10], [11]. A primary form of fractional lower-order statistics is covariation defined in (2) which plays a role analogous to autocorrelation. It has been proved that covariation is highly robust to impulsive noise [12]. But in many applications a more convenient FLOS base on covariation are widely used called fractional lower-order correlation

$$
R_{s}^{C}(\tau) \square E\left[s(t+\tau) s^{<p-1>}(t)\right], 1<p \leq 2
$$

In equation (18), the convention $z^{<k>}$ can be expressed by $z^{<k>}=|z|^{k-1} z^{*}$. The fractional lower-order correlation is a robust type of moment, $E\left[s(t+\tau) s^{<p-1>}(t)\right]<\infty$ in the presence of impulsive noise. Moreover, $z^{<k>}$ restrains magnitude but maintains period of random variable $z$. The fractional lower-order correlation $R_{s}^{C}(\tau)$ contains the same period as that of the conventional autocorrelation function. By taking Fourier transform of fractional lower-order correlation, we define a new type of cyclic statistics

$R_{s}^{\varepsilon, C}(\tau) \square\left\langle s(t+\tau) s^{<p-1>}(t) e^{-j 2 \pi \varepsilon t}\right\rangle, 1<p \leq 2$

which called cyclic covariation. The cyclic covariation spectrum is defined to be the Fourier transform of cyclic covariation

$S_{s}^{\varepsilon, C}(f) \square \int_{-\infty}^{\infty} R_{s}^{\varepsilon, C}(\tau) e^{-j 2 \pi f \tau} d \tau$

It follows from (19) and (20) that for $p=2$, the fractional lower-order cyclic covariation $R_{s}^{\varepsilon, C}(\tau)$ and covariation spectrum $S_{s}^{\varepsilon, C}(f)$ reduce to the second-order cyclic autocorrelation $R_{s}^{\varepsilon}(\tau)$ and cyclic spectrum $S_{s}^{\varepsilon}(\tau)$. For cycle frequency $\varepsilon=0, R_{s}^{\varepsilon, C}(\tau)$ and $S_{s}^{\varepsilon, C}(f)$ become the conventional $R_{s}^{C}(\tau)$ and generalized covariation spectrum. Therefore, the cyclic covariation and cyclic covariation spectrum presented in this paper make use of the benefits of both fractional lower-order statistics and cyclic statistics. The proposed $R_{s}^{\varepsilon, C}(\tau)$ and $S_{s}^{\varepsilon, C}(f)$ is applicable even in the presence of impulsive noise. They have the ability to exploit spectral correlation property of cyclostationary signals and robust against impulsive noise. The performance of conventional cyclic matched filter degrades due to the unboundness of cyclostationarity in $S \alpha S$ impulsive noise.

In order to enhance the performance of cyclic matched filter, we formulate a new cyclic matched filtering method by utilizing fractional lower-order cyclostationarity instead of second-order cyclic statistics. Substituting (1) into (19), $R_{x}^{\varepsilon, C}(\tau)$ can be obtained

$R_{x}^{\varepsilon, C}(\tau)=R_{s}^{\varepsilon, C}(\tau)+R_{n s}^{\varepsilon, C}(\tau)$

We assume that $z_{x}^{C}(\tau)=R_{x}^{\varepsilon, C}(\tau), z_{s}^{C}(\tau)=R_{s}^{\varepsilon, C}(\tau)$, and $m^{C}(\tau)=R_{n s}^{\varepsilon, C}(\tau)$, then equation (21) yields

$z_{x}^{C}(\tau)=z_{s}^{C}(\tau)+m^{C}(\tau)$

where $z_{s}^{C}(\tau)$ is the received signal, $m^{C}(\tau)$ is interference and noise. Given this model, $z_{s}^{C}(\tau)$ can be detected from $z_{x}^{C}(\tau)$ using the linear matched filter which based on the max-output-SNR criterion, where the impulse response $h_{s}^{C}(\tau)$ of matched filter can be obtained when the max output SNR is achieved at $t=\tau_{0}$. We assume $S_{z_{s}}^{C}(f)$ is the spectrum of $z_{s}^{C}(\tau), H^{C}(f)$ is the transfer function of the filter, the output signal to noise power ratio at $t=\tau_{0}$ is given by

$d_{\tau_{0}} \square \frac{\left|\int_{-\infty}^{\infty} H^{C}(f) S_{z_{s}}^{C}(f) e^{j 2 \pi f \tau_{0}} d f\right|^{2}}{\int_{-\infty}^{\infty}\left|H^{C}(f)\right|^{2} G_{n}^{C}(f) d f}$

where 
$G_{n}^{C}(f)=\left|\int(n(t))^{<p-1>} e^{-j 2 \pi f t} d t\right|^{2}$

From the Cauchy-Schwarz inequality, we can see that

$d_{\tau_{0}} \leq \int_{-\infty}^{\infty} \frac{\left|S_{z_{s}}^{C}(f)\right|^{2}}{G_{n}^{C}(f)} d f$

If and only if $H^{C}(f) \sqrt{G_{n}^{C}(f)} e^{j 2 \pi f \tau_{0}}=c\left(\frac{S_{z_{s}}^{C}(f)}{\sqrt{G_{n}^{C}(f)}}\right)^{*}$, can the equality in (25) hold. Then, the transfer function is given by

$H^{C}(f)=c \frac{\left(S_{z_{s}}^{C}(f)\right)^{*}}{G_{n}^{C}(f)} e^{j 2 \pi f \tau_{0}}$

From equation (21) we find that

$S_{z_{s}}^{C}(f)=S_{s}^{\varepsilon, C}(f)$

Substitution of (27) into (26) will yield

$H^{C}(f)=c \frac{\left(S_{s}^{\varepsilon, C}(f)\right)^{*}}{G_{n}^{C}(f)} e^{j 2 \pi f \tau_{0}}$

The fractional lower-order cyclic matched filter has improved performance compare with the classical cyclic matched filter. It can deemphasize the effects of impulsive noise effectively, and exhibits signal-selectivity property. In real world application, the cyclic covariation spectrum $S_{s}^{\varepsilon, C}(f)$ of the signal $s(t)$ can not be computed directly, we can use cyclic covariation spectrum $S_{x}^{\varepsilon, C}(f)$ as the estimation of $S_{s}^{\varepsilon, C}(f)$. Furthermore, the fractional lower-order cyclic matched filter can reduce to the cyclic matched filter when $p=2$. It reduces to the fractional lower-order matched filter, when cycle frequency $\varepsilon=0$. When cycle frequency $\varepsilon=0$ and $p=2$, the fractional lower-order cyclic matched filter reduces to the classical matched filter. Therefore, fractional lower-order cyclic matched filter can operate properly in detection of cyclostationary signals in the presence of interfering signals, Gaussian noise, and alpha-stable impulsive noise.

As explained in [5], typical cycle frequency in these signals include the keying rate, and its harmonics in keyed digital systems, and doubled carrier frequency in continuous-wave analog systems, as well as sums and differences of these cycle frequencies in digital carrier-modulated systems. Since the cycle frequency for most communication signals are not unique, multiple single-cycle estimators can be fused together to form a multi-cycle matched filter bank in highly corruptive interference environment to outperform the drawbacks of single-cycle estimators. In addition, the proposed method is a class of detectors parameterized by $p$, include the conventional cyclic matched filter $(p=2)$ and matched filter $(p=2, \varepsilon=0)$ as special cases. Note that the characteristic exponent $\alpha$ of stable noise is usually unknown. It can be estimated from the data, and the parameter employed in (28) can be chosen as $p<\hat{\alpha} / 2$ in practice.

\section{Simulation Results}

In this section, we present the comparative results on performance of the new fractional lower-order cyclic matched filter versus cyclic matched filter. The signal of interest is a real simulated BPSK signal with uncorrelated $S \alpha S$ noise. The carrier frequency of the BPSK signal is $f_{c}=0.2 / T_{s}$, keying rate of $\varepsilon_{k}=0.0625 / T_{s}$. The discrete time sampling increment is $T_{s}$ with the integration time $T=8192 T_{s}$. As the stable distribution makes the standard SNR meaningless, we use generalized signal-to-noise ratio (GSNR) as the ratio of the signal power over the impulsive noise dispersion $\gamma$, GSNR $=10 \log _{10}\left(E\left[|s(t)|^{2}\right] / \gamma\right)[8]$.

\section{A. Effects of impulsive noise on cyclostationarity}

The cyclic spectrum and cyclic covariation spectrum of BPSK signal which has carrier frequency $f=0.2 f_{s}$ and keying rate $\varepsilon=0.0625 f_{s}$ in the presence of Gaussian noise ( $\alpha=2$ ) and alpha-stable impulsive noise ( $\alpha=1.8$ ) are shown in Fig. 1. The GSNR is $0 \mathrm{~dB}$. It can be seen from Fig. 1(a) and (c) that, the conventional cyclic spectrum and the cyclic covariation spectrum exhibit the similar performance in Gaussian noise, they are immune to Gaussian noise. However, Fig. 1(b) shows that the conventional cyclic spectrum is masked severely in impulsive noise, the cyclostationarity of BPSK signal can not be distinguished. Compared with cyclic spectrum the cyclic covariation spectrum reveals a highly suppression capability of impulsive noise

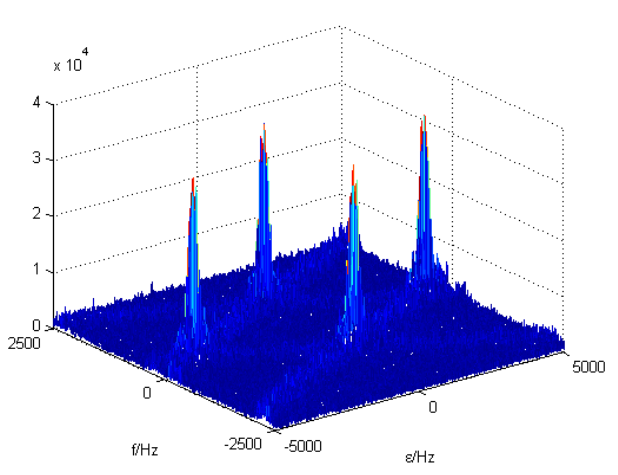

(a)

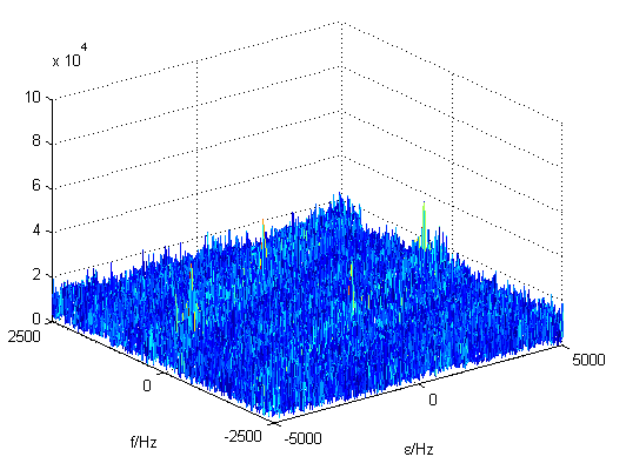

(b) 


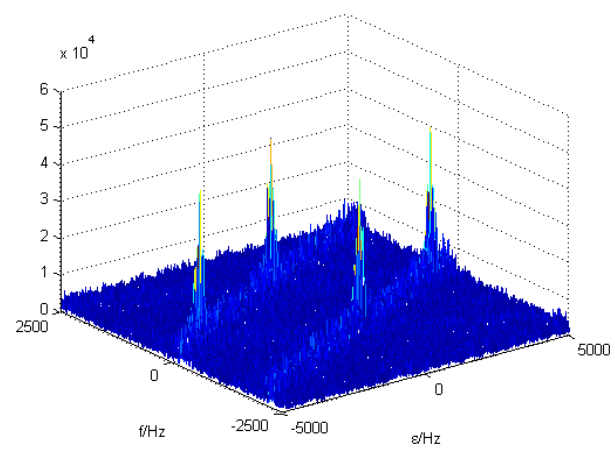

(c)

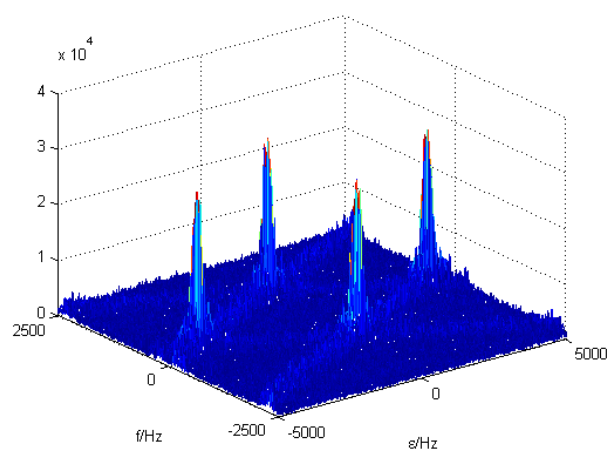

(d)

Fig. 1 Effects of impulsive noise on cyclostationarity

(a) Cyclic spectrum in Gaussian noise. (b) Cyclic spectrum in impulsive noise. (c) Cyclic covariation spectrum in Gaussian noise. (d) Cyclic covariation spectrum in impulsive noise

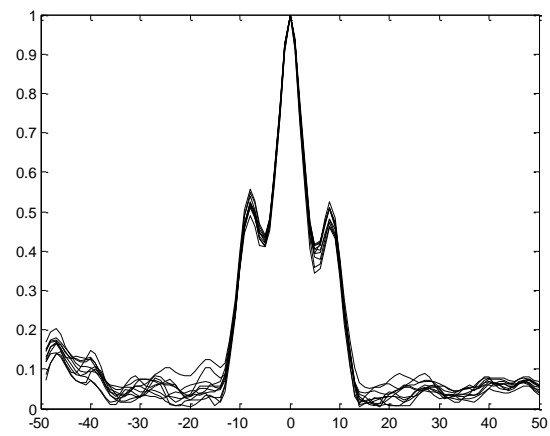

(a)

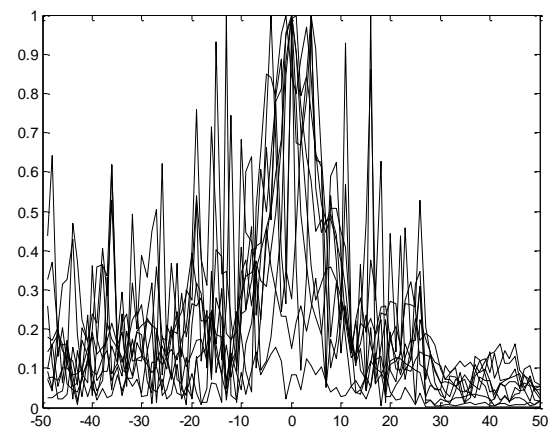

(b)

Fig.2 Outputs of the cyclic matched filter(a) Gaussian noise (b) Impulsive noise

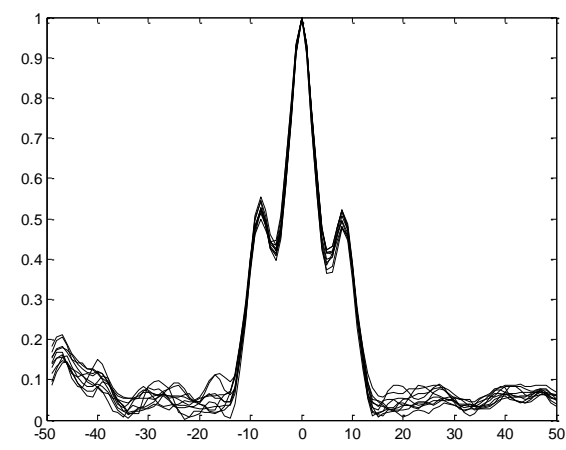

(a)

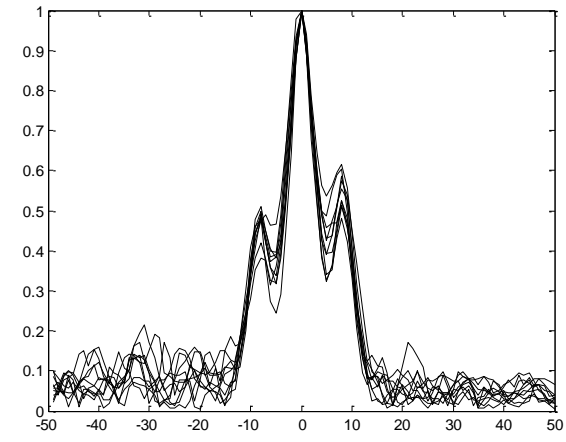

(b)

Fig.3 Outputs of the robust cyclic matched filter(a) Gaussian noise (b) Impulsive noise

\section{B. Effects of impulsive noise on cyclic matched filter}

The exponent characteristic of alpha-stable process is 2 , and GSNR is $0 \mathrm{~dB}$. The outputs of conventional cyclic matched filters of ten independent simulations in the presence of Gaussian noise $(\alpha=2)$ and impulsive noise $(\alpha=1.8)$ are shown in Fig. 2. Fig. 3 shows the results of the new robust cyclic matched filters in the presence of Gaussian noise $(p=1.2)$ and impulsive noise $(p=1.2)$.

\section{Effects of interference on the conventional and proposed approaches}

In this case, the interference [4] consists of five AM signals with carrier frequencies of $f_{1}=0.156 / T_{s}, f_{2}=0.203 / T_{s}$, $f_{3}=0.266 / T_{s}, f_{4}=0.313 / T_{s}$, and $f_{5}=0.375 / T_{s}$, with bandwidths of $B_{1}=0.04 / T_{s}, B_{2}=0.05 / T_{s}, B_{3}=0.045 / T_{s}$, $B_{4}=0.04 / T_{s}$, and $B_{5}=0.08 / T_{s}$. The signal-to-interference ratio (SIR) of each AM signal is $0 \mathrm{~dB}$ and GSNR is $0 \mathrm{~dB}$, which yields a total signal-to-noise ratio (SNR) of $-8 \mathrm{~dB}$. The characteristic exponent of $S \alpha S$ noise is $\alpha=1.6$. The cycle frequencies exploited by the algorithms are $0, \varepsilon=0.0625 / T_{s}$, $2 \varepsilon$, and $2 f_{c}$. Outputs of the proposed robust cyclic matched filter using different cycle frequencies from ten independent simulations are shown in Fig.4. Simulation results show that $2 f_{c}$ detector is superior to the other detectors. It is that the cycle 


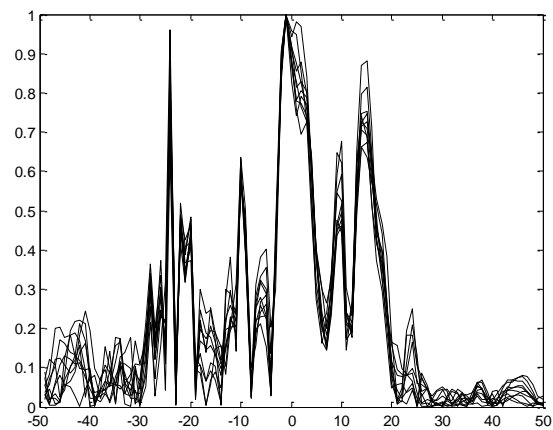

(a)

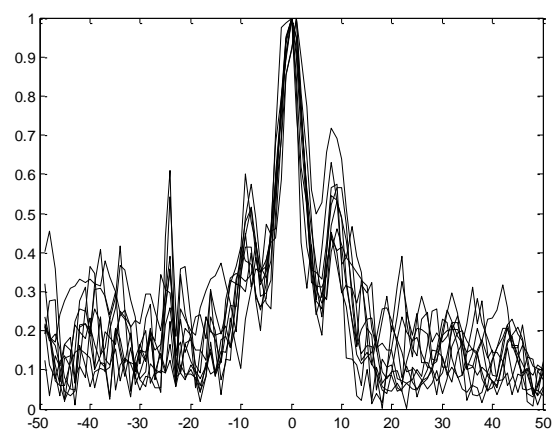

(b)

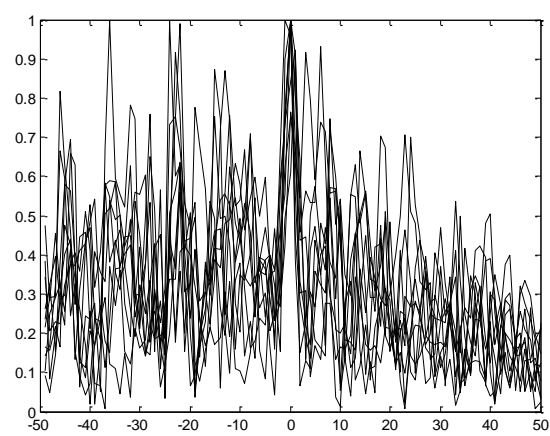

(c)

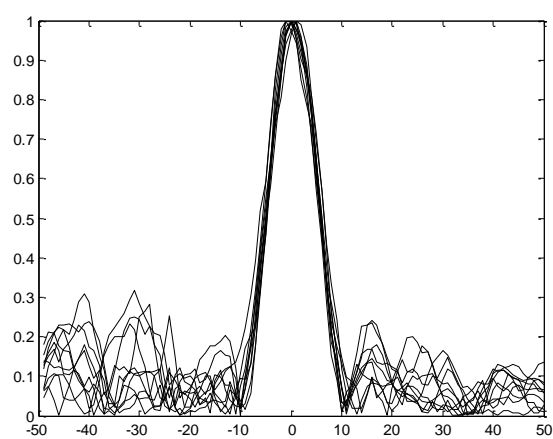

(d)

Fig.4 Outputs of the robust cyclic matched filter (a) $\varepsilon=0$ (b) $\varepsilon=\varepsilon_{k}$ (c) $\varepsilon=2 \varepsilon_{k}$ (d) $\varepsilon=2 f_{c}$

frequency $\varepsilon=2 f_{c}$ is typically stronger than that at $\varepsilon=\varepsilon_{k}, \varepsilon=2 \varepsilon_{k}$.

Detection performance for the matched filter (ML), the fractional lower-order covariance based matched filter

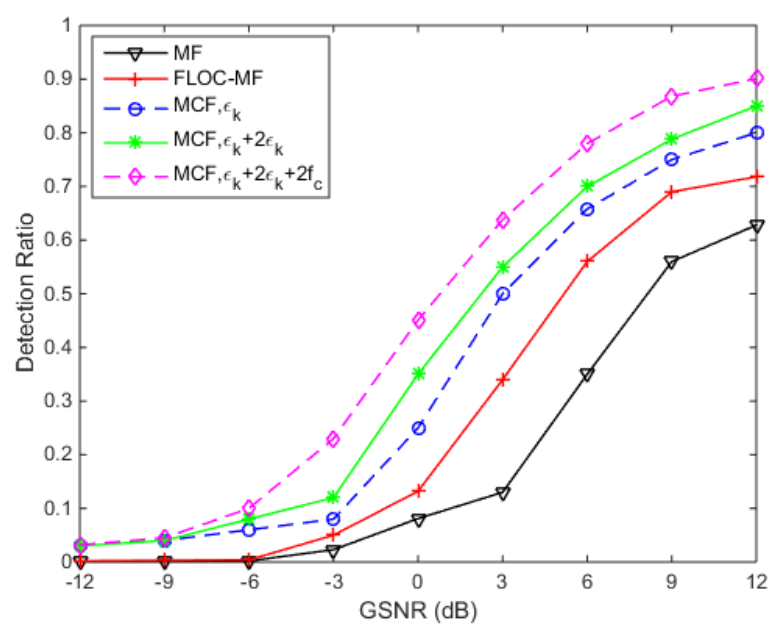

(a)

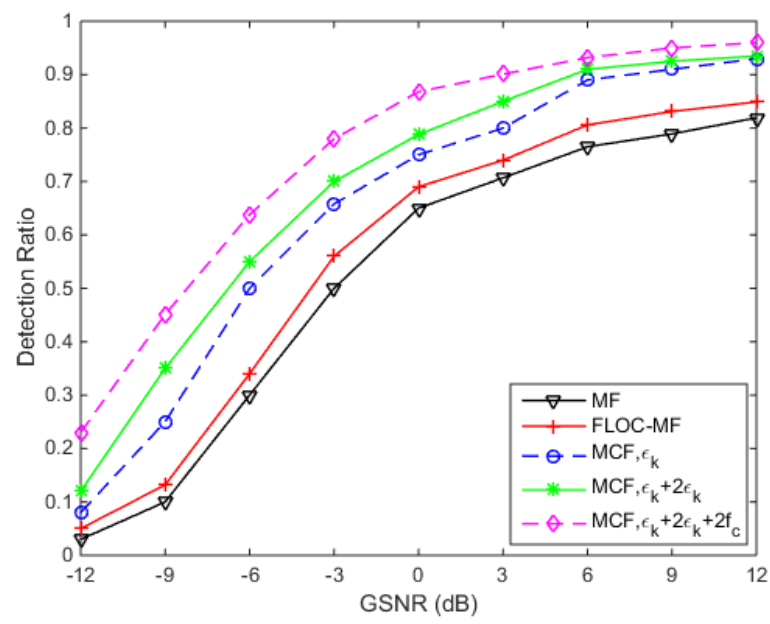

(b)

Fig.5 Performance of different detection algorithms in impulsive noise (a) $\alpha=1.6$ (b) $\alpha=1.8$.

(FLOC-ML) and the proposed robust multi cycle frequency (MCF) detector in the highly impulsive noise $(\alpha=1.6)$ and slightly impulsive noise ( $\alpha=1.8$ ) are shown in Fig.5. It can be seen from simulation results that the impulsive characteristic is an important factor for the performance of all algorithms, because the detection ratio for all the algorithms in the case of $\alpha=1.6$ is inferior to the case of $\alpha=1.8$. However, the fractional lower-order statistics based FLOC-MF and the proposed algorithm based on the fractional lower-order cyclostationarity are more robust to the impulsive noise than the second order statistics based matched filter. In addition, since the AM interferences are present, the performance of the proposed cyclic algorithms outperforms the FLOC-MF and MF. Moreover, it is clear that the multi cycle frequencies can improve the performance. Because the cyclostationarity of the signal of interest is stronger in $\varepsilon=2 f_{c}$ than in $\varepsilon=\varepsilon_{k}$ and $\varepsilon=2 \varepsilon_{k}$, thus, from Fig.5(a) and Fig.5(b) we can see that performance of the double cycle frequencies detector is superior to the single cycle frequency detector, but is inferior to the triple cycle frequencies detector. 


\section{CONCLUSION}

In this paper, we study the representation of cyclostationarity and TDOA estimation methods for cyclostationary signals in the presence of interfering signals and heavy-tailed $\alpha$-stable impulsive noise. Conventional second-order cyclostationarity exploiting methods perform poorly in impulsive noise environments. We propose two types of representations for revealing the cyclostationarity property of signals using PFLOM operation. We introduce two classes of TDOA estimation algorithms based on fractional lower-order cyclostationarity. The new methods parameterized by PFLOM exponent make better use of the cyclostationarity property and FLOS. The performance of the proposed estimation methods is examined in simulations. Simulation results demonstrate the effectiveness and robustness of the new representations and algorithms. It is shown that the performance of the proposed algorithms is significantly better than the conventional estimators in a wide range of interference and noise environments.

\section{REFERENCES}

[1] A. T. Schonhoff, A. A. Giordano, "Detection and estimation theory and its application", Prentice hall, New Jersey, 2007.

[2] A. Glover, P. M. Grant, "Digital communications", Pearson hall, New York, 2004.

[3] D. Ramírez, P. J. Schreier, J. Vía, I. Santamaría, L. L. Scharf, "Detection of multivariate cyclostationarity”, IEEE Trans. Signal Process., vol.60, No.2, 2015, pp. 5395-5408.

[4] A. Napolitano, "Cyclostationarity: Limits and generalizations. Signal Process", Signal Process., vol.120, No.3, 2016, pp. 323-347.

[5] W. A. Gardner, A. Napolitano, L. Paura, "Cyclostationarity: Half a century of research", Signal Process., vol.86, No.4, 2006, pp. 639-697.

[6] A. Napolitano, "Generalizations of cyclostationarity: A new paradigm for signal processing for mobile communications, radar, and sonar", IEEE Signal Process. Mag., vol.30, No.6, 2013, pp.53-63.

[7] W. A. Gardner, "Cyclic wiener filtering: Theory and methods", IEEE Trans. Commun., vol.41, No.1, 1993, pp. 151-163.

[8] Z. Huang, Y. Zhou, W. Jiang, "On cyclic correlation matched filtering", Acta Electron. Sin., vol.30, No.12, 2002, pp. 122-126.

[9] M. Shao, C. L. Nikias, "Signal processing with Alpha-stable distributions and fractional lower order moments: stable process and their applications", Proceedings of the IEEE, vol.81, No.7, 1993, pp. 986-1010.

[10] C. L. Nikias, M. Shao, "Signal processing with Alpha-Stable Distributions and Applications", Wiley, New York, 1995.

[11] T. Bai, H. Zhang, J. Zhang, et al., "Impulsive noise mitigation in digital subscriber lines: The state-of-the-art and research opportunities", IEEE Commun. Mag., vol.57, No.5, 2019, pp. 145-151.

[12] K. Hassan, R. Gautier, I. Dayoub, et al., "Multiple antenna based blind spectrum sensing in the presence of impulsive noise", IEEE Trans. Veh. Technol., vol.63, No.5, 2014, pp. 2248-2257.

[13] Y. F. Chen, J. M. Chen, "Novel SS PDF approximations and their applications in wireless signal detection”, IEEE Trans. Wireless Commun., vol.14, No.2, 2015, pp. 1080-1091.

[14] F. X. Socheleau, D. Pastor, M. Duret, "On symmetric alpha-stable noise after short time Fourier transformation", IEEE Signal Process. Lett., vol.20, No.5, 2013, pp. 455-458

[15] A. Vannucci, G. Colavolpe, L. Veltri, et al., "Estimation of correlated Gaussian samples in impulsive noise", IEEE Commun. Lett., vol.24, No.1, 2020, pp. 103-107.

[16] A. M. Wilson, T. Panigrahi, A. Dubey, "Robust distributed Lorentzian adaptive filter with diffusion strategy in impulsive noise environment", Digital Signal Process., vol.96, 2020, pp. 1-13.

[17] S. Cambanis, G. Miller, "Linear problem in pth order and stable process", SIAM J. Appl. Math, vol.41, No.1, 1981, pp. 43-69.
[18] R. Barazideh, B. Natarajan, A. V. Nikitin, et al., "Performance analysis of analog intermittently nonlinear filter in the presence of impulsive noise", IEEE Trans. Veh. Technol., vol.68, No.4, 2019, pp. 3565-3573.

[19] S. Niranjayan, N. C. Beaulieu, "The BER optical linear rake receiver for signal detection in symmetric alpha-stable noise", IEEE Trans. Commun., vol.57, No.12, 2009, pp. 3585-3588.

[20] H. S. Lim, T. C. Chuah, H. T. Chuah, "Robust chip-matched myriad filter-based multiuser receiver for impulsive channels", Signal Process., vol.88, No.5, 2008, pp. 1216-1232.

[21] J. G. Gonzalez, J. L. Paredes, G. R. Arce, "Zero-Order Statistics: a mathematical framework for the processing and characterization of very impulsive signals", IEEE Trans. Signal Process., vol.54, No.10, 2006, pp. 3839-3851

\section{Creative Commons Attribution License 4.0 (Attribution 4.0 International, CC BY 4.0)}

This article is published under the terms of the Creative Commons Attribution License 4.0

https://creativecommons.org/licenses/by/4.0/deed.en_US 